IJMMS 29:5 (2002) 303-306

PII. S0161171202003071

http://ijmms.hindawi.com

(c) Hindawi Publishing Corp.

\title{
SOME SEQUENCE SPACES AND STATISTICAL CONVERGENCE
}

\section{E. SAVAŞ}

\author{
Received 29 January 1999
}

We introduce the strongly $(V, \lambda)$-convergent sequences and give the relation between strongly $(V, \lambda)$-convergence and strongly $(V, \lambda)$-convergence with respect to a modulus. 2000 Mathematics Subject Classification: 40D25, 40A05, 40C05.

1. Introduction. Let $\lambda=\left(\lambda_{n}\right)$ be a nondecreasing sequence of positive numbers tending to $\infty$, and $\lambda_{n+1} \leq \lambda_{n}+1, \lambda_{1}=1$.

The generalized de la Vallée-Poussin mean is defined by

$$
t_{n}=\frac{1}{\lambda_{n}} \sum_{k \in I_{n}} x_{k}
$$

where $I_{n}=\left[n-\lambda_{n}+1, n\right]$. A sequence $x=\left(x_{k}\right)$ is said to be $(V, \lambda)$-summable to a number $L$ (see [5]) if $t_{n}(x) \rightarrow L$ as $n \rightarrow \infty$. If $\lambda_{n}=n$, then $(V, \lambda)$-summability is reduced to $(C, 1)$-summability. We write

$$
[V, \lambda]=\left\{x=\left(x_{k}\right): \text { for some } L, \lim _{n} \frac{1}{\lambda_{n}} \sum_{k \in I_{n}}\left|x_{k}-L\right|=0\right\}
$$

for sets of sequences $x=\left(x_{k}\right)$ which are strongly $(V, \lambda)$-summable to $L$, that is, $x_{k} \rightarrow$ $L[V, \lambda]$.

We recall that a modulus $f$ is a function from $[0, \infty)$ to $[0, \infty)$ such that

(i) $f(x)=0$ if and only if $x=0$;

(ii) $f(x+y) \leq f(x)+f(y)$ for all $x, y \geq 0$;

(iii) $f$ is increasing;

(iv) $f$ is continuous from the right at 0 .

It follows that $f$ must be continuous on $[0, \infty)$. A modulus may be bounded or unbounded. Maddox [6] and Ruckle [9] used the modulus $f$ to construct sequence spaces. In this paper, we introduce the strongly $(V, \lambda)$-convergent sequences and give the relation between strongly $(V, \lambda)$-convergence and strongly $(V, \lambda)$-convergence with respect to a modulus.

\section{Some sequence spaces}

Definition 2.1. Let $f$ be a modulus. We define the spaces,

$$
\begin{aligned}
{[V, \lambda, f] } & =\left\{x=\left(x_{k}\right): \lim _{n} \frac{1}{\lambda_{n}} \sum_{k \in I_{n}} f\left(\left|x_{k}-L\right|\right)=0, \text { for some } L\right\}, \\
{[V, \lambda, f]_{0} } & =\left\{x=\left(x_{k}\right): \lim _{n} \frac{1}{\lambda_{n}} \sum_{k \in I_{n}} f\left(\left|x_{k}\right|\right)=0\right\} .
\end{aligned}
$$


When $\lambda_{n}=n$ then the sequence spaces defined above become $w_{0}(f)$ and $w(f)$, respectively, where $w_{0}(f)$ and $w(f)$ are defined by Maddox [6].

Note that if we put $f(x)=x$, then we have $[V, \lambda, f]=[V, \lambda]$ and $[V, \lambda, f]_{0}=[V, \lambda]_{0}$, where

$$
[V, \lambda]_{0}=\left\{x=\left(x_{k}\right): \lim _{n} \frac{1}{\lambda_{n}} \sum_{k \in I_{n}}\left|x_{k}\right|=0\right\} .
$$

We have the following result.

THEOREM 2.2. The spaces $[V, \lambda, f]$ and $[V, \lambda, f]_{0}$ are linear spaces.

Proof. We consider only $[V, \lambda, f]$. Suppose that $x_{i} \rightarrow L$ and $y_{j} \rightarrow L^{\prime}$ in $[V, \lambda, f]$ and that $\alpha, \beta$ are in $\mathbb{C}$. Then there exists integers $T_{\alpha}$ and $M_{\beta}$ such that $|\alpha| \leq T_{\alpha}$ and $|\beta| \leq M_{\beta}$. We therefore have

$$
\begin{aligned}
& \frac{1}{\lambda_{n}} \sum_{k \in I_{n}} f\left(\left|\alpha x_{k}+\beta x_{k}-\left(\alpha L+\beta L^{\prime}\right)\right|\right) \\
& \quad \leq T_{\alpha} \frac{1}{\lambda_{n}} \sum_{k \in I_{n}} f\left(\left|x_{k}-L\right|\right)+M_{\beta} \frac{1}{\lambda_{n}} \sum_{k \in I_{n}} f\left(\left|x_{k}-L^{\prime}\right|\right)
\end{aligned}
$$

This implies that $\alpha x+\beta y \rightarrow \alpha L+\beta L^{\prime}$ in $[V, \lambda, f]$. This completes the proof.

Proposition 2.3 (see [7]). Let $f$ be any modulus. Then $\lim _{t \rightarrow \infty} f(t) / t=\beta$ exists.

Proposition 2.4. Let $f$ be a modulus and let $0<\delta<1$. Then for each $x \geq \delta$ we have $f(x) \leq 2 f(1) \delta^{-1} x$.

This can be proved by using the techniques similar to those used in Maddox [6] and hence we omit the proof.

THEOREM 2.5. Let $f$ be any modulus. If $\lim _{t \rightarrow \infty} f(t) / t=\beta>0$, then $[V, \lambda, f]=[V, \lambda]$.

Proof. If $x \in[V, \lambda]$, then

$$
s_{n}=\frac{1}{\lambda_{n}} \sum_{k \in I_{n}}\left|x_{k}-L\right| \longrightarrow 0 \text { as } n \longrightarrow \infty \text {, for some } L .
$$

Let $\varepsilon>0$ and choose $\delta$ with $0<\delta<1$ such that $f(t)<\varepsilon$ for every $t$ with $0 \leq t \leq \delta$. We can write

$$
\begin{aligned}
\frac{1}{\lambda_{n}} \sum_{k \in I_{n}} f\left(\left|x_{k}-L\right|\right) & =\frac{1}{\lambda_{n}} \sum_{k \in I_{n},\left|x_{k}-L\right| \leq \delta} f\left(\left|x_{k}-L\right|\right)+\frac{1}{\lambda_{n}} \sum_{k \in I_{n},\left|x_{k}-L\right|>\delta} f\left(\left|x_{k}-L\right|\right) \\
& \leq \frac{1}{\lambda_{n}}\left(\lambda_{n} \cdot \varepsilon\right)+2 f(1) \delta^{-1} s_{n}
\end{aligned}
$$

by Proposition 2.4, as $n \rightarrow \infty$. Therefore $x \in[V, \lambda, f]$. It is trivial that $[V, \lambda, f] \subset[V, \lambda]$ and this completes the proof. 
3. $\lambda$-statistical convergence. In [3], Fast introduced the idea of statistical convergence, which is closely related to the concept of natural density or asymptotic density of subsets of the positive integers $\mathbb{N}$. In recent years, statistical convergence has been studied by several authors $[1,2,4,8,10]$.

A sequence $x=\left(x_{k}\right)$ is said to be statistically convergent to the number $L$ if for every $\varepsilon>0$,

$$
\lim _{n} \frac{1}{n}\left|\left\{k \leq n:\left|x_{k}-L\right| \geq \varepsilon\right\}\right|=0
$$

where the vertical bars indicate the number of elements in the enclosed set. In this case we write $s-\lim x=L$ or $x_{k} \rightarrow L(s)$ and $s$ denotes the set of all statistically convergent sequences.

In this section, we introduce and study the concept of $\lambda$-statistical convergence and find its relation with $[V, \lambda, f]$ and $s_{\lambda}$.

DEFINITION 3.1. A sequence $x=\left(x_{k}\right)$ is said to be $\lambda$-statistically convergent or $s_{\lambda}$-convergent to $L$ if for every $\varepsilon>0$,

$$
\lim _{n} \frac{1}{\lambda_{n}}\left|\left\{k \in I_{n}:\left|x_{k}-L\right| \geq \varepsilon\right\}\right|=0
$$

In this case, we write $s_{\lambda}-\lim x=L$ or $x_{k} \rightarrow L\left(s_{\lambda}\right)$ and $s_{\lambda}=\left\{x\right.$ : for some $L, s_{\lambda}-$ $\lim x=L\}$. Note that if $\lambda_{n}=n$, then $s_{\lambda}$ is same as $s$.

The following definition was introduced by Connor [2] as an extension of the original definition of statistical convergence which appeared in [3].

DEFINITION 3.2. Let $A$ be a nonnegative regular summability method and let $x$ be a sequence. Then $x$ is said to be $A$-statistically convergent to $L$ if $\chi_{S(x-L e: \varepsilon)}$ is contained in $w_{0}(A)$ for every $\varepsilon>0$, where

$$
w_{0}(A)=\left\{x: \lim _{n} \sum a_{n, k}\left|x_{k}\right|=0\right\} .
$$

In the above definition, if we define the matrix by

$$
a_{n, k}= \begin{cases}\frac{1}{\lambda_{n}}, & \text { if } n \in I_{n}, \\ 0, & \text { if } n \notin I_{n}\end{cases}
$$

we get $\lambda$-statistical convergence as a special case of $A$-statistical convergence.

Let $\nabla$ denote the set of all nondecreasing sequences $\lambda=\left(\lambda_{n}\right)$ of positive numbers tending to $\infty$ such that $\lambda_{n+1} \leq \lambda_{n}+1$ and $\lambda_{1}=1$.

We have the following result.

THEOREM 3.3. Let $\lambda \in \nabla$ and $f$ be any modulus. Then $[V, \lambda, f] \subset\left(s_{\lambda}\right)$. 
Proof. Suppose that $\varepsilon>0$ and $x \in[V, \lambda, f]$. Since,

$$
\begin{aligned}
\frac{1}{\lambda_{n}} \sum_{k \in I_{n}} f\left(\left|x_{k}-L\right|\right) & \geq \frac{1}{\lambda_{n}} \sum_{k \in I_{n},\left|x_{k}-L\right| \geq \varepsilon} f\left(\left|x_{k}-L\right|\right) \\
& \geq \frac{1}{\lambda_{n}} f(\varepsilon) \cdot\left|\left\{k \in I_{n}:\left|x_{k}-L\right| \geq \varepsilon\right\}\right|
\end{aligned}
$$

from which it follows that $x \in\left(s_{\lambda}\right)$. This completes the proof.

THEOREM 3.4. $\left(s_{\lambda}\right)=[V, \lambda, f]$ if and only if $f$ is bounded.

Proof. Suppose that $f$ is bounded and that $x \in\left(s_{\lambda}\right)$. Since $f$ is bounded, there is a constant $M$ such that $f(x) \leq M$ for all $x \geq 0$. Given $\varepsilon>0$, we have

$$
\begin{aligned}
\frac{1}{\lambda_{n}} \sum_{k \in I_{n}} f\left(\left|x_{k}-L\right|\right) & \leq \frac{1}{\lambda_{n}} \sum_{k \in I_{n},\left|x_{k}-L\right| \geq \varepsilon} f\left(\left|x_{k}-L\right|\right)+\frac{1}{\lambda_{n}} \sum_{k \in I_{n},\left|x_{k}-L\right|<\varepsilon} f\left(\left|x_{k}-L\right|\right) \\
& \leq \frac{M}{\lambda_{n}}\left|\left\{k \in I_{n}:\left|x_{k}-L\right| \geq \varepsilon\right\}\right|+f(\varepsilon) .
\end{aligned}
$$

Taking the limit as $\varepsilon \rightarrow 0$, the result follows. Conversely, suppose that $f$ is unbounded so that there is a positive sequence $0<t_{1}<t_{2}<\cdots<t_{i}<\cdots$ such that $f\left(t_{i}\right) \geq \lambda_{i}$. Define the sequence $x=\left(x_{i}\right)$ by putting $x_{k_{i}}=t_{i}$ for $i=1,2, \ldots$ and $x_{i}=0$ otherwise. Then we have $x \in\left(s_{\lambda}\right)$, but $x \notin[V, \lambda, f]$.

\section{REFERENCES}

[1] J. Connor, The statistical and strong p-Cesàro convergence of sequences, Analysis 8 (1988), no. 1-2, 47-63.

[2] _ On strong matrix summability with respect to a modulus and statistical convergence, Canad. Math. Bull. 32 (1989), no. 2, 194-198.

[3] H. Fast, Sur la convergence statistique, Colloq. Math. 2 (1951), 241-244 (1952) (French).

[4] J. A. Fridy, On statistical convergence, Analysis 5 (1985), no. 4, 301-313.

[5] L. Leindler, Über die verallgemeinerte de la Vallée-Poussinsche Summierbarkeit allgemeiner Orthogonalreihen, Acta Math. Acad. Sci. Hungar. 16 (1965), 375-387 (German).

[6] I. J. Maddox, Sequence spaces defined by a modulus, Math. Proc. Cambridge Philos. Soc. 100 (1986), no. 1, 161-166.

[7] _ Inclusions between FK spaces and Kuttner's theorem, Math. Proc. Cambridge Philos. Soc. 101 (1987), no. 3, 523-527.

[8] D. Rath and B. C. Tripathy, On statistically convergent and statistically Cauchy sequences, Indian J. Pure Appl. Math. 25 (1994), no. 4, 381-386.

[9] W. H. Ruckle, FK spaces in which the sequence of coordinate vectors is bounded, Canad. J. Math. 25 (1973), 973-978.

[10] T. Šalát, On statistically convergent sequences of real numbers, Math. Slovaca 30 (1980), no. $2,139-150$.

E. SAVAŞ: DePARTMENT OF MATHEMATICS, YÜZÜNCÜ YIL UNIVERSITY, VAN, TURKeY

E-mail address: ekremsavas@yahoo.com 


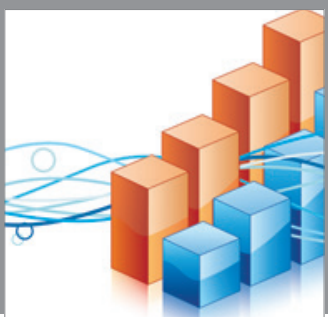

Advances in

Operations Research

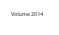

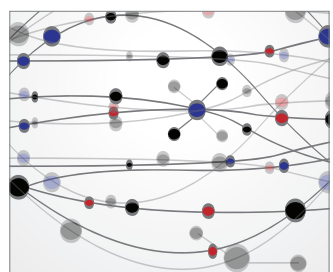

\section{The Scientific} World Journal
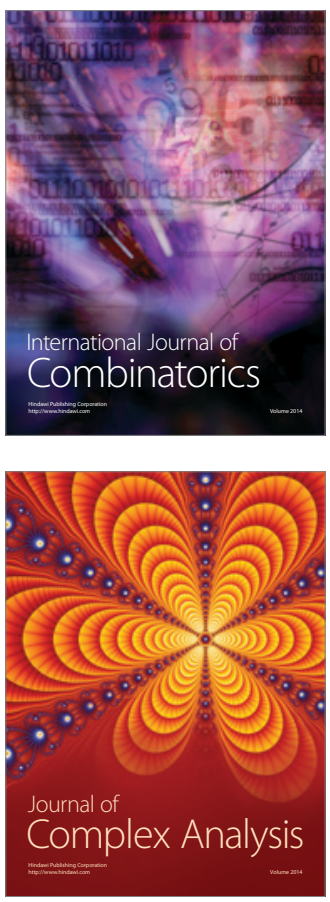

International Journal of

Mathematics and

Mathematical

Sciences
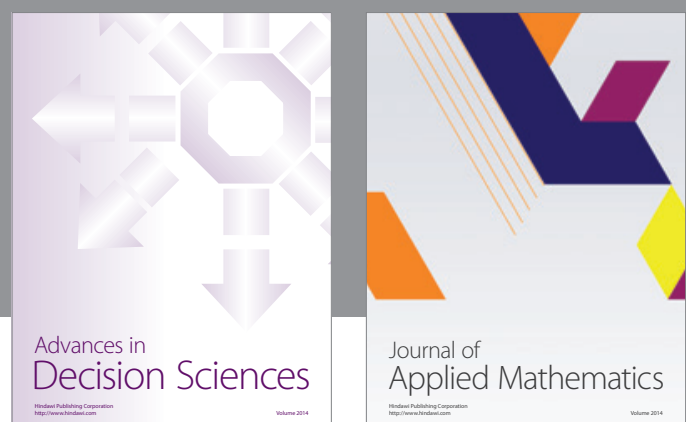

Journal of

Applied Mathematics
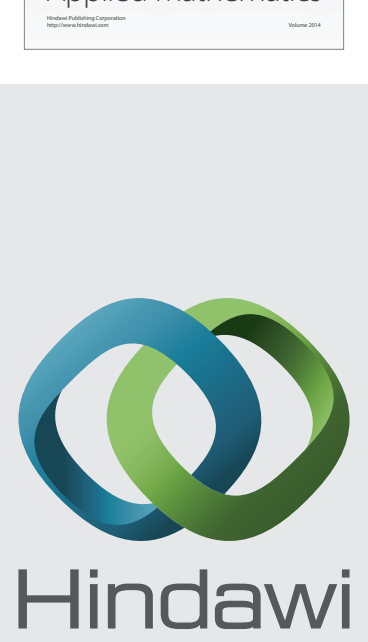

Submit your manuscripts at http://www.hindawi.com
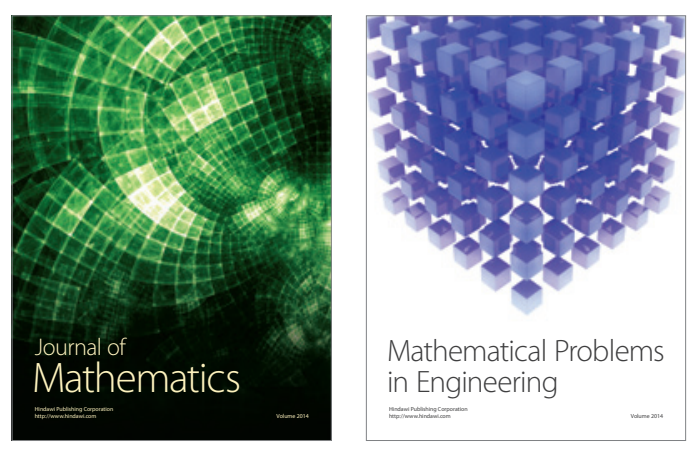

Mathematical Problems in Engineering
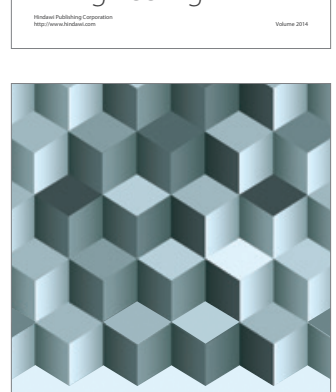

Journal of

Function Spaces
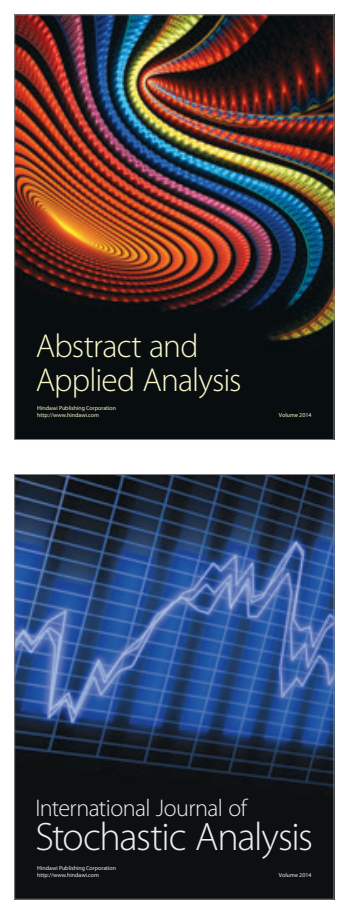

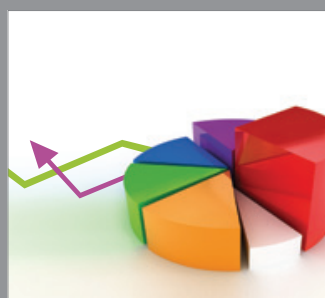

ournal of

Probability and Statistics

Promensencen
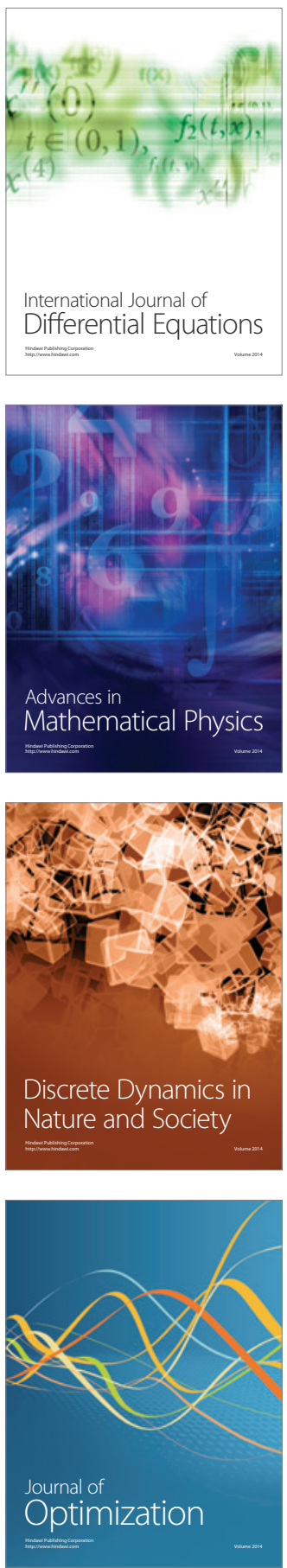